\title{
Effect of Inhomogeneous Mixture Properties on CI Combustion in a Schnurle-Type Gasoline DI Engine*
}

\author{
Seok-woo KIM** and Yasuo MORIYOSHI ${ }^{* * *}$
}

\begin{abstract}
The authors have performed experiments on compression-ignition (CI) for a singlecylinder Schnurle-type two-stroke gasoline direct injection (DI) engine which employs a variable exhaust port, area, and deduced two presumptions from the experimental results. Firstly, the spatial distributions of fuel concentration and in-cylinder gas temperature are indispensable to enable $\mathrm{CI}$ operation under stratified charge conditions, because $\mathrm{CI}$ operation is not possible in a DI system although the necessary conditions of the scavenging efficiency and the in-cylinder gas temperature for the initiation of $\mathrm{CI}$ in homogeneous charge conditions are satisfied. Secondly, it is possible that flame propagation occurs in stratified charge CI conditions, because the combustion period in the later stage after $80 \%$ mass burned becomes longer than that with homogeneous charge CI combustion. In this report, in order to verify the above two presumptions deduced from experiments, the gas exchange process and mixture formation process were numerically analyzed, and the initiation conditions of CI were estimated using a CHEMKIN application. As a result, in case of CI with a late injection timing in DI system, it was found that CI was possible because high temperature but no fuel region and low temperature but rich fuel region exist in the cylinder due to inhomogeneous spatial distributions of fuel and temperature. Also, in case of CI with a late injection timing, the flame propagation was possible in the low-temperature and diluted rich region. Thereby, the two presumptions deduced from the experimental results were validated from the numerical analysis results.
\end{abstract}

Key Words: Ignition, Fuel Injection, Gasoline Engine, Stratified Flow, Internal Combustion Engine, CI Engine, Inhomogeneous Charge

\section{Introduction}

The authors have proposed a new concept for a small two-stroke gasoline engine in their previous report ${ }^{(1)}$; the optimized performance can be achieved by homogeneous SI (spark-ignition) combustion at high loads, homogeneous CI (CI) combustion at medium loads and stratified CI combustion at low loads. In the previous report, the CI combustion characteristics at medium loads and speeds were examined; the effects of the fuel supply method on the performance of CI combustion and the possibility to control the ignition timing by varying the injection timing

${ }^{*}$ Received 27th April, 2005 (No. 05-4030)

** Graduate school of Science and Technology, Chiba University, 1-33 Yayoi-cho, Inage-ku, Chiba 263-8522, Japan. E-mail: kimsw@graduate.chiba-u.jp

*** Department of Electronics and Mechanical Engineering, Chiba University, 1-33 Yayoi-cho, Inage-ku, Chiba 2638522, Japan. E-mail: ymoriyos@ faculty.chiba-u.jp for direct injection conditions. As a result, the operational region where $\mathrm{CI}$ combustion can be achieved was found almost the same between the port injection system and DI system with early injection, and BMEP value was almost equal regardless of the type of injection, while BSFC and BSHC were much lower in DI system.

Also, in case of medium loads at $4000 \mathrm{r} / \mathrm{min}$, it was found that when the scavenging efficiency was lower than $70 \%$ and the estimated gas temperature at TDC was higher than $1180 \mathrm{~K}, \mathrm{CI}$ operation was possible. However, CI operation was not possible for some cases in DI conditions although the above necessary conditions of the scavenging efficiency and the in-cylinder gas temperature were satisfied. This means that other conditions such as the spatial distributions of fuel concentration and temperature inside the cylinder are indispensable to enable CI operation in the direct injection system.

Furthermore, in case of stratified CI combustion with a late DI timing, the combustion period in the later stage of 
combustion period after $80 \%$ mass burned became longer than that with a port injection. For this reason, it was estimated that combustion was performed by flame propagation in a portion with a diluted rich or stoichiometric mixture and low temperature where $\mathrm{CI}$ combustion was impossible.

In this research, in order to verify the abovementioned two presumptions, the gas exchange process and mixture formation process in a Schnurle-type gasoline DI engine were numerically analyzed, and the initiation conditions of CI were estimated using a CHEMKIN application.

\section{Experimental Apparatus}

\subsection{Test engine}

Calculations were carried out in a water-cooled single-cylinder Schnurle-type two-stroke gasoline engine (HONDA-CRM250AR) modified to enable the incylinder direct fuel injection. Major engine specifications are listed in Table 1. The calculation meshes at BDC are shown in Fig. 1. The number of calculation grids is $39 \times 97 \times 28$, while the total number of the mesh is about 114000 .

The exhaust valve located at the exhaust port was used to adjust the amount of the internal EGR (Exhaust Gas Recirculation). The amount of the internal EGR was

Table 1 Engine specifications

\begin{tabular}{|c||c|}
\hline Engine Type & 2-Stroke Single Cylinder \\
\hline Fuel Supply & Direct $/$ Port Injection \\
\hline Bore $\times$ Stroke & $\phi 66.4 \times 72.0 \mathrm{~mm}$ \\
\hline Displacement & $250 \mathrm{cc}$ \\
\hline Effective C.R. & 6.7 \\
\hline Exh. Port Open & $90 \mathrm{deg}$. ATDC \\
Close & $270 \mathrm{deg}$. ATDC \\
\hline Scav. Port Open & $120 \mathrm{deg}$. ATDC \\
Close & $240 \mathrm{deg}$. ATDC \\
\hline
\end{tabular}

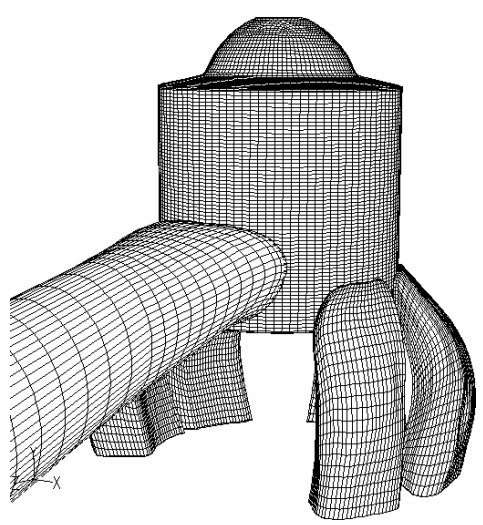

Fig. 1 Calculation mesh at BDC increased with a decreased exhaust port area by closing the exhaust valve. The VAR (Valve Angle Rate of the exhaust valve), which was parametrically varied, is shown in Fig. 2. As shown in Fig. 2, the exhaust port area was adjusted in the calculation by setting some meshes as "wall". The VAR $=0 \%$ shown in Fig. 2 (b) means the full-closed exhaust valve in the base-engine with the exhaust port area of $3 \mathrm{~cm}^{2}$.

\section{Calculation Methods}

\subsection{In-cylinder numerical analysis}

Numerical analysis conditions are listed in Table 2. Engine speed, VAR, Injection timing (IT) as shown in Table 2 were parametrically varied, while the gas exchange process, mixture formation process, and spray behavior were numerically analyzed using a general-purpose thermofluids code of Fluent (Ver.4.5).

As the basic equations, N-S Eqs., continuity Eqs., enthalpy Eqs., gas law, k- $\varepsilon$ Eqs. as turbulence model, and transport Eqs. of two gas species (burned gas and fresh air) were solved implicitly ${ }^{(3)}$. As the spray model, Discrete Droplet Model $^{(2)}$ (DDM) was used. Because DDM was not implemented in Fluent4.5, DDM was added as a user sub-routine, and then the calculation was performed. Furthermore, as sub-models for DDM, a swirl injector model $^{(4)}$, a wall collision model, a breakup model ${ }^{(5)-(7)}$ and a vaporization model were added. Regarding the parameters of the breakup model, optimized valves by the authors were used ${ }^{(8)}$.

The initial and boundary conditions of the calcula-

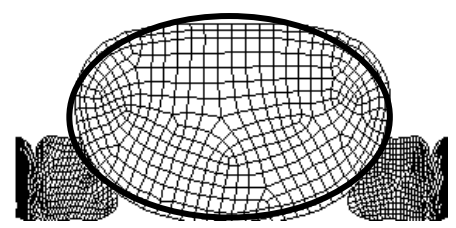

(a) VAR $=100 \%$

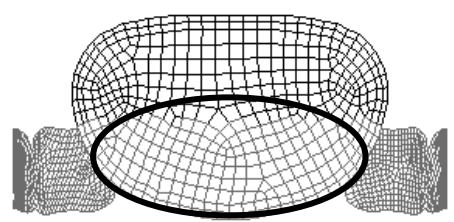

(b) $\mathrm{VAR}=0 \%$

Fig. 2 Exhaust port mesh at different VAR

Table 2 Calculation conditions

\begin{tabular}{|c|c|c|c|}
\hline Case & $\begin{array}{c}\text { Engine Speed } \\
{[\mathrm{r} / \mathrm{min}]}\end{array}$ & $\begin{array}{l}\text { VAR } \\
{[\%]} \\
\end{array}$ & $\begin{array}{l}\text { Injection Timing } \\
\text { [deg.ATDC] }\end{array}$ \\
\hline Cal-1 & \multirow{4}{*}{4000} & \multirow{2}{*}{0} & 180 \\
\hline Cal-2 & & & 270 \\
\hline Cal-3 & & \multirow{2}{*}{50} & 180 \\
\hline Cal-4 & & & 270 \\
\hline
\end{tabular}


Table 3 Injection conditions

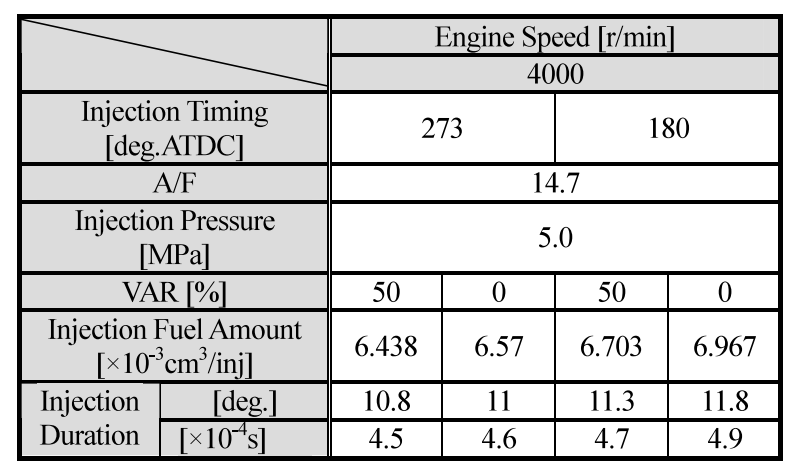

Table 4 Boundary conditions $(\phi=1.0)$

\begin{tabular}{|c||c|c|}
\hline & $\begin{array}{c}\text { Scavenging Port } \\
\text { Inlet }\end{array}$ & $\begin{array}{c}\text { Exhaust Port } \\
\text { Outlet }\end{array}$ \\
\hline Pressure & Measured & $101 \mathrm{kPa}$ \\
\hline Temperature [K] & \multicolumn{2}{|c|}{300} \\
\hline Turbulence Intensity & $10 \%$ of mean piston speed \\
\hline $\begin{array}{c}\text { Turbulence Length } \\
\text { Scale [m] }\end{array}$ & 0.002 \\
\hline & Wall \\
\hline Temperature $[\mathrm{K}]$ & 500 \\
\hline
\end{tabular}

tion are shown in Tables 3 and 4, respectively. The measured pressure values at in-cylinder, exhaust and scavenging ports, were used as the initial and boundary conditions for the calculation ${ }^{(1)}$. Temperature was assumed using the gas law, and iso-octane was used as a fuel.

The calculations were carried out from 90 deg.ATDC to 360 deg.ATDC and combustion was not performed.

\subsection{Estimation of CI initiation}

Occurrence status and initiation timing of CI were estimated using the $\operatorname{SENKIN}^{(9)}$ application included in CHEMKIN 2.0 on zero-dimensional, homogeneous and adiabatic condition. For iso-octane, the mechanism consists of 857 species and 3606 reactions $^{(10)}$. The initial values were given from the results of 3D mixture formation calculation at the end of injection.

Firstly, calculations were conducted at homogeneous condition with $4000 \mathrm{r} / \mathrm{min}, \mathrm{VAR}=0 \%$ and $50 \%$. The initial values are listed in Table 5. Moreover, calculations were conducted at heterogeneous conditions using a special technique mentioned later. A comparison between the two calculation results will be discussed.

SENKIN application was used to estimate the initiation timing of $\mathrm{CI}$ in homogeneous condition. In order to estimate the initiation timing of $\mathrm{CI}$ in heterogeneous condition, the result of $3 \mathrm{D}$ numerical analysis at 280 deg.ATDC was used as the initial value, and calculations were sequentially conducted in every $20 \mathrm{deg}$. to TDC with an assumption of quasi-steady in each $20 \mathrm{deg}$. For the initial values of SENKIN, in-cylinder temperature and
Table 5 Initial values in homogeneous conditions $(\phi=1.0)$

\begin{tabular}{|c|c|c|c|c|c|c|}
\hline $\begin{array}{c}\text { Engine } \\
\text { Speed } \\
{[\mathrm{r} / \mathrm{min}]}\end{array}$ & $\begin{array}{c}\text { VAR } \\
{[\%]}\end{array}$ & $\begin{array}{c}\text { IT } \\
{[\text { deg.ATDC] }}\end{array}$ & $\begin{array}{c}\text { Start C.A. } \\
{[\text { [deg.ATDC }]}\end{array}$ & $\begin{array}{c}\text { Tint } \\
{[\mathrm{K}]}\end{array}$ & $\begin{array}{c}\text { Pint } \\
{[\mathrm{kPa}]}\end{array}$ & $\begin{array}{c}\text { EGR } \\
{[\%]}\end{array}$ \\
\hline \multirow{3}{*}{4000} & \multirow{2}{*}{0} & 180 & 255 & 307 & 110 & 32 \\
\cline { 2 - 7 } & \multirow{2}{*}{50} & 270 & 290 & 612 & 218 & 39 \\
\cline { 2 - 7 } & 180 & 255 & 430 & 120 & 58 \\
\cline { 3 - 7 } & & 270 & 290 & 721 & 216 & 60 \\
\hline
\end{tabular}

IT: Injection Timing, Tint: Initial Temperature, Pint: Initial Pressure

Table 6 Initial values in heterogeneous conditions ( $4000 \mathrm{r} / \mathrm{min}$, $\mathrm{VAR}=0 \%, \mathrm{IT}=270$ deg. ATDC)

\begin{tabular}{|c|c|c|c|c|c|c|c|c|c|c|}
\hline \multirow{2}{*}{$\begin{array}{c}\text { VAR } \\
\text { [\%] }\end{array}$} & $\begin{array}{c}\text { C.A. } \\
\text { [deg.ATDC] }\end{array}$ & \multicolumn{3}{|c|}{ Point A } & \multicolumn{4}{c|}{ Point B } & \multicolumn{3}{c|}{ Point C } \\
\cline { 2 - 12 } & $\phi$ & Temp & EGR & $\phi$ & Temp & EGR & $\phi$ & Temp & EGR \\
\hline \multirow{4}{*}{0} & 280 & 0 & 739 & 90 & 0.01 & 722 & 88 & 2.24 & 653 & 83.5 \\
\cline { 2 - 12 } & 300 & 0.61 & 758 & 88.7 & 1.30 & 747 & 87.2 & 2.23 & 708 & 86.2 \\
\cline { 2 - 11 } & 320 & 1.06 & 973 & 91.1 & 1.77 & 966 & 90.4 & 5.74 & 841 & 81.8 \\
\cline { 2 - 11 } & 340 & 0.60 & 1200 & 93.7 & 1.10 & 1200 & 93.3 & 8.24 & 978 & 79.5 \\
\cline { 2 - 10 } & 360 & 0.46 & 1330 & 94.9 & 0.91 & 1330 & 94.7 & 8.88 & 1100 & 80.7 \\
\hline
\end{tabular}

Table 7 Initial values in heterogeneous conditions (4 000 r/min, $\mathrm{VAR}=50 \%, \mathrm{IT}=270 \mathrm{deg} . \mathrm{ATDC})$

\begin{tabular}{|c|c|c|c|c|c|c|c|c|c|c|}
\hline \multirow{2}{*}{$\begin{array}{l}\text { VAR } \\
{[\%]}\end{array}$} & \multirow{2}{*}{$\begin{array}{c}\text { C.A. } \\
\text { [deg.ATDC] }\end{array}$} & \multicolumn{3}{|c|}{ Point A } & \multicolumn{3}{|c|}{ Point B } & \multicolumn{3}{|c|}{ Point C } \\
\hline & & $\phi$ & Temp & EGR & $\phi$ & Temp & EGR & $\phi$ & Temp & EGR \\
\hline \multirow{5}{*}{50} & 280 & 0 & 570 & 61.8 & 0.51 & 612 & 81.9 & 0.12 & 564 & 72 \\
\hline & 300 & 0 & 718 & 74.8 & 2.87 & 689 & 82.2 & 0.88 & 623 & 70 \\
\hline & 320 & 0.1 & 901 & 85.7 & 1.79 & 837 & 85 & 3.87 & 702 & 65.3 \\
\hline & 340 & 0.56 & 1110 & 90 & 1.32 & 1020 & 85 & 5.33 & 827 & 64 \\
\hline & 360 & 0.7 & 1200 & 91 & 3.19 & 1120 & 84.5 & 7.49 & 961 & 70 \\
\hline
\end{tabular}

pressure were given from the result of SENKIN calculation in the previous step, while EGR and equivalence ratio were given from the results of Fluent calculation. Hereinafter, this method will be called discretization SENKIN method.

In case of $4000 \mathrm{r} / \mathrm{min}, \quad \mathrm{VAR}=0 \%$, IT $=270$ deg.ATDC and VAR $=50 \%$, the initial values of discretization SENKIN method are shown in Tables 6 and 7, respectively. Points A, B and C in the table respectively indicate the portion with high-temperature and lean-mixture, medium-temperature and nearly stoichiometric, and low-temperature and rich-mixture.

\section{Results and Discussions}

\subsection{Effect of injection timing}

Numerical analysis were performed at Cal-1 and 2. The spatial distributions of the in-cylinder evaporated fuel mass fraction and the in-cylinder temperature are shown in Fig. 3.

Regarding the evaporated fuel, in case of the injection timing at $270 \mathrm{deg}$.ATDC, much difference of the evaporated fuel occurs as shown in Fig. 3 (a), therefore, a heterogeneous distribution of the in-cylinder mixture is apparent. However, in case of the injection timing at $180 \mathrm{deg}$.ATDC, relatively little difference of the evaporated fuel is shown in Fig. 3 (b). Regarding the in-cylinder gas temperature, in case of the injection timing at $270 \mathrm{deg}$.ATDC, due to 


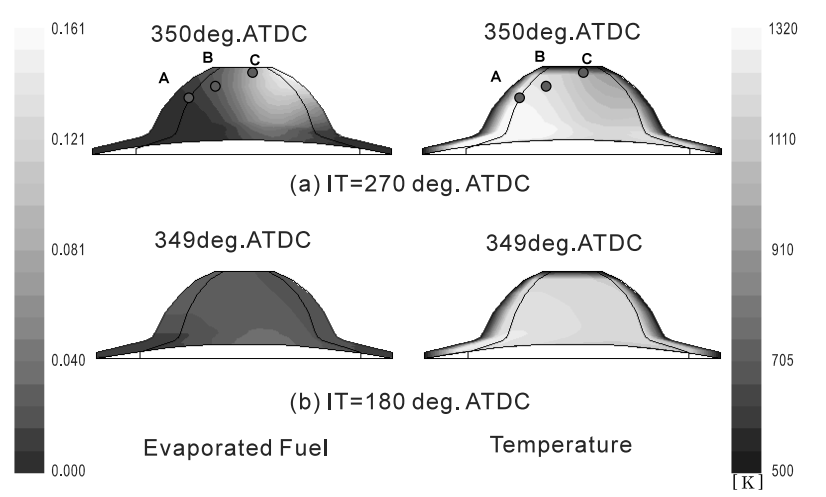

Fig. 3 Spatial distributions of evaporated fuel and temperature at the initiation timing of combustion $(4000 \mathrm{r} / \mathrm{min}$, $\operatorname{VAR}=0 \%)$

heterogeneous distributions of the in-cylinder gas temperature and fuel concentration, in-cylinder gas temperature is distributed in a wide range of $950-1300 \mathrm{~K}$, and the masimum gas temperature is approximately $50-70 \mathrm{~K}$ higher than that at $180 \mathrm{deg}$.ATDC. On the other hand, in case of IT $=180 \mathrm{deg}$. ATDC, a homogeneous distributions of the in-cylinder gas and fuel concentration are formed. Thereby, the in-cylinder gas temperature is distributed narrowly in the range of $1150-1250 \mathrm{~K}$ and the maximum in-cylinder gas temperature is lower than that of 270 deg.ATDC.

However, the region of high temperature is larger in comparison with the injection timing at $270 \mathrm{deg}$.ATDC, leading to a great advantage to satisfy the temperature condition for CI. Actually, in case of the same experimental condition, $\mathrm{CI}$ occurs in both the injection timings at 270 and 180 deg.ATDC.

In case of CI with direct injection in the experiments ${ }^{(1)}$, when the CI occurs, the in-cylinder gas temperature is above $1053 \mathrm{~K}$ at $4000 \mathrm{r} / \mathrm{min}$. Therefore, the condition to cause $\mathrm{CI}$ is satisfied in both the injection timings at 270 and $180 \mathrm{deg}$.ATDC, as compared with the calculation result shown in Fig. 3. In particular, in case of the injection timing at $180 \mathrm{deg}$.ATDC, the combustion period becomes shorter and the variation of pressure becomes lower because there is a portion of high temperature in a wide region. In case of the injection timing at $270 \mathrm{deg}$.ATDC, the condition for attaining CI early because there is a portion of high temperature than that of $180 \mathrm{deg}$.ATDC in the same crank angle.

Figure 4 shows the spatial distributions of the incylinder fuel mass fraction and the in-cylinder temperature at the initiation timing of $\mathrm{CI}$ from the results of numerical analysis at Cal-1 and 2.

The spatial distributions of the evaporated fuel and the temperature indicate the same tendency as shown in Fig. 3. The mixture becomes heterogeneous as the injection timing is set late. In case of VAR $=50 \%$, IT $=270$ deg.ATDC, because of the inhomogeneity of the

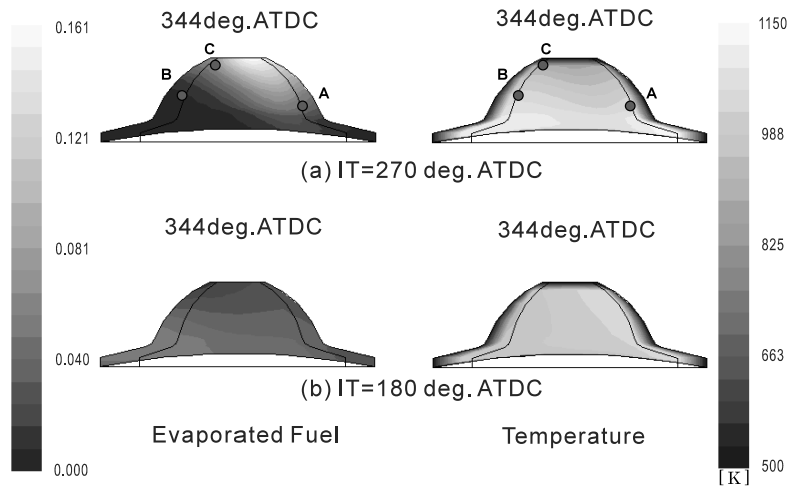

Fig. 4 Spatial distributions of evaporated fuel and temperature at the initiation timing of combustion $(4000 \mathrm{r} / \mathrm{min}$, $\operatorname{VAR}=50 \%$ )

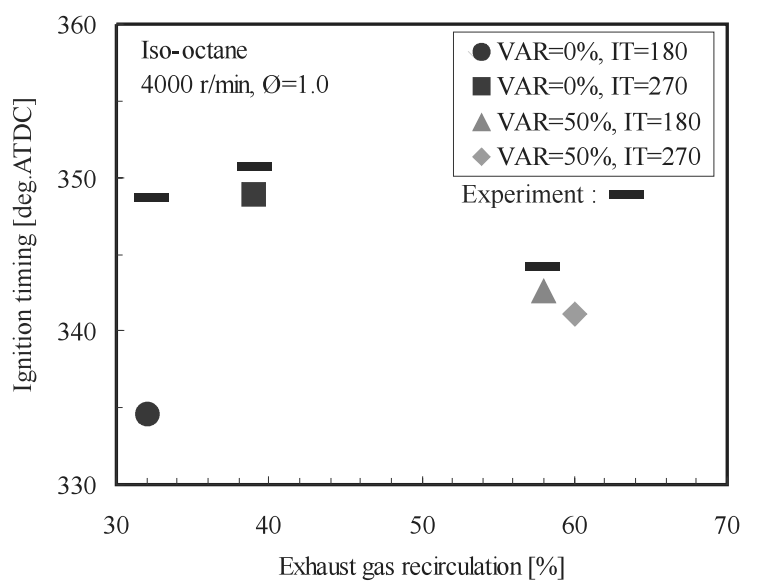

Fig. 5 Ignition timing vs EGR rate in homogeneous conditions

in-cylinder mixture and the temperature, a region with a higher temperature than of IT $=180 \mathrm{deg}$.ATDC exists, but, a rich vapor is not distributed. Moreover even in the portion of higher fuel concentration, since the temperature is below $900 \mathrm{~K}, \mathrm{CI}$ is impossible. According to the experimental result, CI was possible at IT $=180 \mathrm{deg}$. ATDC, while it was impossible at IT $=270 \mathrm{deg}$.ATDC although the gas temperature and scavenging efficiency conditions for CI deduced from homogeneously charged experiments were satisfied.

In conclusion, another condition for CI, such as the spatial distributions of the fuel concentration and the incylinder temperature is indispensable to enable CI operation in direct injection system was found indispensable.

\subsection{Estimation of CI initiation by SENKIN}

Figure 5 shows the predicted results of the initiation status of CI, the initiation timing of CI from Cal-1 to Cal4.

CI was predicted possible in all conditions, also the initiation timing of CI was almost the same at $\mathrm{VAR}=0 \%$, 270 deg. ATDC and VAR $=50 \%, 180$ deg.ATDC. However, the initiation timing of CI was predicted earlier in comparison with experimental results. In particular, in 
Table 8 Ignition timing in heterogeneous conditions at $4000 \mathrm{r} / \mathrm{m}$

\begin{tabular}{|c|c|c|c|c|c|}
\hline Engine & \multirow{2}{*}{$\begin{array}{c}\text { Speed } \\
\text { [r/min] }\end{array}$} & VAR & IT & \multicolumn{4}{|c|}{ Ignition Timing } \\
\cline { 4 - 6 } & {$[$ deg. ATDC] } & Point A & Point B & Point C \\
\hline \multirow{2}{*}{4000} & 0 & \multirow{2}{*}{270} & 350.2 & 350.9 & $\mathrm{X}$ \\
\cline { 5 - 7 } & 50 & & 354.8 & $\mathrm{X}$ & $\mathrm{X}$ \\
\hline
\end{tabular}

case of VAR $=50 \%, 270$ deg.ATDC, CI was predicted possible, while CI was impossible in the experiments. This is because the spatial distributions of temperature and fuel concentration are inhomogeneous by means of later injection timing, as shown in Figs. 3 and 4.

Here, the calculation results of heterogeneous condition at Cal-2 and 4 by means of discretization SENKIN method are shown in Table 8. In case of Cal-2, CI occurs at points $\mathrm{A}$ and $\mathrm{B}$, but not at point $\mathrm{C}$. This is probably due to that the temperature was not satisfied with the initiation condition of $\mathrm{CI}$ although rich vapor was distributed at point $\mathrm{C}$. Also, at point $\mathrm{C}$, it was found that flame propagation was possible, because the local equivalence ratio was within the flammable condition ${ }^{(11)}$. Thus, CI occurs at points $\mathrm{A}$ and $\mathrm{B}$, and then the flame can be propagated to point $\mathrm{C}^{(12)}$.

Meanwhile, in the experiment of the same condition $^{(1)}$, the later stage of combustion after $80 \%$ mass burned fraction becomes slow regardless of an engine speed. For this reason, the authors presumed that the stratification of the in-cylinder mixture resulting from direct injection caused $\mathrm{CI}$ at a portion with rich mixture and high temperature, while only flame propagation occurred in a portion with cold and diluted flammable mixture. The calculated result in Table 8 indicates that flammable mixture in low gas temperature exists at points $\mathrm{B}$ and $\mathrm{C}$ while there is a lean mixture in high temperature at point $\mathrm{A}$. Thereby, CI occurs around point $\mathrm{A}$ in rapid combustion speed and then the flame propagates towards points B and C. This simulation result verifies the author's presumptions. Hence, presumptions from the experiments were evaluated theoretically by the calculation results.

\section{Conclusions}

A calculation mesh of a water-cooled single-cylinder Schnurle-type two-stroke gasoline engine with a variable exhaust port area was made in order to carry out numerical analyses using commercial codes of Fluent and CHEMKIN at the medium load and medium speed $(4000 \mathrm{r} / \mathrm{min}$, throttle opening rate of $20 \%$, equivalence rate of 1.0). Two presumptions deduced from the experimental results were validated from the numerical analysis results.

( 1 ) In case of late injection timing with DI system, CI was not possible although the necessary conditions, which were established from experimental results with port injection system, were satisfied. For this reason, it was found from both experiments and calculations that high temperature but no enough fuel region and low temperature but rich fuel region exist in the cylinder due to inhomogeneous spatial distributions of fuel and temperature. This means that the spatial distributions of fuel concentration and temperature must be taken in account in DI systems.

( 2 ) In case of CI with late injection timings, the combustion period in the later stage of combustion period after $80 \%$ mass burned became long. For this reason, it was found that low-temperature but diluted rich region exists in the cylinder due to inhomogeneous distributions of fuel and temperature. CI was not possible in the lowtemperature and diluted rich region, but flame propagation was possible. Thus, CI occurs at high-temperature and lean region, and then the flame propagates to lowtemperature and diluted rich region with relatively slow combustion speed regardless of the engine speed.

(3) It is difficult to estimate the initiation timing of CI in inhomogeneous conditions using SENKIN, since SENKIN application originally computes the time evolution of a homogeneous reacting mixture. However, the initiation timing of CI could be estimated in inhomogeneous conditions by using discretization SENKIN method developed by the authors, by combining with the 3D flow calculation results. This tool was found useful to make evaluations of both the experimental and theoretical results.

\section{Acknowledgements}

The authors would like to thank Prof. T. Kitagawa at Kyusyu Univ. and Prof. K. Tanoue at Oita Univ. who gave useful information to use SENKIN application, and also the related companies who supplied mechanical parts and measurement devices.

\section{References}

( 1 ) Kim, S.W. and Moriyoshi, Y., Analysis of CI Combustion in a Schnurle-Type Gasoline Engine, KSME International Journal, Vol.18, No.8 (2004), pp.1473-1482.

(2) Moriyoshi, Y., Takaki, M. and Hu, X., Analysis of Mixture Formation Process with a Swirl-Type Injector, SAE Paper, No.2000-01-2057, (2000), pp.1-11.

( 3 ) Fluent Inc., Fluent User's Guide, Lebanon, Vol.1-4 (1996).

( 4 ) Han, Z., Modeling Atomization Processes of PressureSwirl Hollow-Cone Fuel Sprays, Atomization and Sprays, Vol.7 (1997), p.663.

( 5 ) Reitz, R.D., Modeling Atomization Processes in HighPressure Vaporizing Sprays, Atomization and Spray Tech., Vol.3 (1987), p.309.

(6) O'Rourke, P.J., The TAB Method for Numerical Calculation of Spray Droplet Breakup, SAE Paper, No.872089. 
( 7 ) Tanagawa, Investigation of Machine, (in Japanese), Vol.15, No.12 (1963), p.1515.

( 8 ) Moriyoshi, Y., Hu, X. and Takagi, M., Experimentally Evaluated Spray Model for a Swirl-Type Injector, SAE Paper, No.2002-01-2696.

(9) Luz, A.E., Kee, R.J. and Miller, J.A., SENKIN:A FORTRAN Program for Predicting Homogeneous Gas Phase Chemical Kinetics with Sensitivity Analysis, Sandia National Laboratory, Report No. SAND878248, (1988).

(10) Curran, H.J.,Gaffuri, P., Pitz, W.J. and Westbrook,
C.K., A Comprehensive Modeling Study of Iso-Octane Oxidation, Combustion and Flame, Vol.129 (2002), pp.253-280.

(11) Hirano, T., Combustion, (in Japanese), (1997), p.113, KAIBUNDO, Tokyo.

(12) Kaneko, M., Morikawa, K., Itoh, J. and Saishu, Y., A Study on Homogeneous Charge Compression Ignition Gasoline Engines Second Report: Control of AutoIgnition by Mixture Stratification, (in Japanese), JSAE Annual Congress, 20015191, (2001). 